\title{
Higher mortality in women after ST-segment elevation myocardial infarction in very young patients
}

Marcin Sadowski ${ }^{1}$, Agnieszka Janion-Sadowska1 ${ }^{1}$, Mariusz Gąsior ${ }^{2}$, Marek Gierlotka², Marianna Janion ${ }^{1,3}$, Lech Poloński

1Świętokrzyskie Cardiology Centre, Kielce, Poland

2Silesian Center for Heart Diseases, Zabrze, Poland

3The University of Humanities and Sciences, Faculty of Health Sciences, Kielce, Poland

Submitted: 7 December 2011

Accepted: 13 March 2012

Arch Med Sci 2013; 9, 3: 427-433

DOI: $10.5114 /$ aoms.2013.35324

Copyright $\odot 2013$ Termedia \& Banach

\begin{abstract}
Introduction: Data on mortality in young patients with ST-segment elevation myocardial infarction (STEMI) when compared to older people or regarding therapeutic strategies are contradictory. We investigate the prognosis of women under 40 after STEMI in a prospective nationwide acute coronary syndrome registry.

Material and methods: We analyzed all 527 consecutive men and women (12.3\% females) aged from 20 to 40 years (mean $35.7 \pm 4.5$ ) presenting with STEMI, of all 26035 STEMI patients enrolled.

Results: Differences between genders in the major cardiovascular risk factors, clinical presentation, extent of the disease and time to reperfusion were insignificant. The majority of patients (67\%) underwent coronary angiography followed by primary percutaneous coronary intervention $(\mathrm{PCI})$ in $79.9 \%$ of them. A $92 \%$ reperfusion success rate measured by post-procedural TIMI 3 flow was achieved. There were no significant differences between genders in the administration of modern pharmacotherapy both on admission and after discharge from hospital. In-hospital mortality was very low in both genders, but 12-month mortality was significantly higher in women $(10.8 \%$ vs. $3.0 \% ; p=0.003)$. Killip class 3 or 4 on admission $(95 \% \mathrm{Cl} 19.6-288.4)$, age per 5 -year increase $(95 \% \mathrm{Cl} 1.01-3.73)$ and primary $\mathrm{PCl}(95 \% \mathrm{Cl} 0.1-0.93)$ affected mortality. In patients who underwent reperfusion there was moderately higher mortality in women than in men (7.1\% vs. $1.9 \% ; p=0.046)$.

Conclusions: Despite little difference in the basic clinical characteristics and the management including a wide use of primary $\mathrm{PCl}$, long-term mortality in women under forty after STEMI is significantly higher than in men.
\end{abstract}

Key words: gender-related difference, ST-segment elevation myocardial infarction, myocardial infarction, mortality, young.

\section{Introduction}

Acute coronary syndromes (ACS) remain one of the leading causes of premature demise in men and women despite unquestionable progress in the management and prevention of coronary artery disease (CAD) [1]. Some classical cardiovascular risk factors, common in the young nowadays, such as smoking, obesity, impaired glucose tolerance, diabetes, dyslipidemia, hyperhomocysteinemia and emotional stress, lead to premature coronary atherosclerosis. Others, being revealed at a younger age and not very prevalent, such as congenital coronary abnormalities, connective
Corresponding author: Marcin Sadowski MD, PhD Świętokrzyskie Cardiology Centre

45 Grunwaldzka St

25-391 Kielce, Poland Phone/fax: +48 413671581 Mobile: +48 606906454

E-mail: emsad@o2.pl 
tissue disorders, increased matrix metalloproteinase activity, coronary artery aneurysm, coronary artery dissection, myocardial bridging, irradiation, illicit drug usage and acquired or congenital hypercoagulability syndromes, may cause an abrupt blood flow cessation due to endothelial dysfunction and coronary artery spasm as well as rapid clot formation $[2,3]$. As myocardial infarction is believed to affect mainly older people, preventive measures are not always a goal in the young. A lot of impressive papers referring to different gender-related issues of ACS have been published so far [4-7]. A female pattern of CAD is related to the high prevalence of non-obstructive eccentric lesions, excessive distal embolization and a tendency toward different structure of the eroded plaque with predominant smooth muscle cells and proteoglycans [8]. Women with STEMI have an increased risk of in-hospital death and their long-term prognosis is worse than their male counterparts $[5,9,10]$; however, there are not many reports on the long-term prognosis in patients with ST-segment elevation myocardial infarction (STEMI) younger than forty. Moreover, in the available literature some contradictory results may be found $[6,9,11]$. Some studies were singlecenter and some mixed different types of acute coronary syndromes.

To further investigate this issue we analyzed a population of 527 consecutive patients aged 20 40 years and their one-year prognosis after STEMI.

\section{Material and methods}

\section{Patients and data collection}

In-hospital data analysis was approved by administrators of the Polish National Acute Coronary Syndrome Registry (PL-ACS), which was carried out by the Silesian Centre for Heart Diseases in Zabrze in cooperation with the National Health Fund as part of the National Program for Prevention and Treatment of Cardiovascular Diseases launched by the Polish Ministry of Health. The principles of the PL-ACS registry have been reported elsewhere [12] and are available on-line [13]. Briefly, the registry includes data on patients with ACS from 456 sites in the country with mandatory data collection. We analyzed all 527 consecutive men and women (12.3\% females) aged from 20 to 40 years (mean $35.7 \pm 4.5$ years) presenting with STEMI, of all 26035 STEMI patients enrolled in the PL-ACS between June 2005 and May 2006. The collected data included age, gender, concomitant diseases, coronary risk factors, previous myocardial infarction and revascularization, time from symptoms onset to admission and reperfusion, extent of the disease in angiography, TIMI flow before and after revascularization, adjunctive treatment, left ventricular ejection fraction (LVEF), complications and medica- tion. Mortality data (in-hospital and 1-year followup) after hospital discharge were obtained from the National Health Fund and Civil Registry database and were collected for all included subjects.

\section{Ethics}

The study was approved by the Ethics Committee of the local division of the Polish Chamber of Physicians.

\section{Statistical analysis}

Continuous variables are expressed as mean \pm standard deviation or median and interquartile range as appropriate. The significance of differences between groups was determined using Student's $t$ test or the Mann-Whitney $U$ test for those variables which did not conform to a normal distribution. Homogeneity of variances was tested using the $F$ test. Categorical variables were tested using the $\chi^{2}$ test. Mortality after 12 months was analyzed using Kaplan-Meier method with a log-rank test. Cox proportional hazards regression was used to adjust mortality for demographic and clinical variables and results are presented as relative risk (RR) and $95 \%$ confidence intervals $(\mathrm{Cl})$. A $p$ value $<0.05$ was considered statistically significant. Calculations and statistical analyses were carried out with Statistica PL software, version 6.1 (StatSoft Inc.).

\section{Results}

\section{Clinical characteristics of the study group}

Of all 26035 patients with STEMI, 2.02\% of those were under forty. There were no significant differences between genders in the basic clinical characteristics (major cardiovascular risk factors, clinical presentation, extent of the disease and time to reperfusion) except for more frequent obesity in women and more frequent moderately decreased left ventricular ejection fraction in men. There was a slight tendency with borderline significance toward more risky cardiovascular profile in women, i.e. diabetes or Killip class on admission (Table I).

\section{Therapeutic approach}

Fibrinolytic treatment was applied in $6.1 \%$ of patients whereas the majority (67\%) underwent coronary angiography followed by primary percutaneous coronary intervention (PCI) in $79.9 \%$ of them (Table II). A 92\% reperfusion success rate measured by post-procedural TIMI 3 flow was achieved (Table III). Women were more likely to receive diuretics ( $15.4 \%$ vs. $7.4 \%$; $p=0.029)$, nitrates ( $25.4 \%$ vs. $14.1 \% ; p=0.02)$, insulin $(10.8 \%$ vs. $3.5 \%$; $p=0.018)$ and oral antihyperglycemic agents $(3.1 \%$ vs. $0.2 \% ; p=0.047$ ). There were no statistically significant differences between genders in the admin- 
Table I. Basic clinical characteristics

\begin{tabular}{|c|c|c|c|}
\hline Variable & Females, $n=65(\%)$ & Males, $n=462(\%)$ & Value of $p$ \\
\hline \multicolumn{4}{|l|}{ Major risk factors } \\
\hline Age [years] & $36.1 \pm 4.0$ & $35.6 \pm 4.6$ & 0.44 \\
\hline Hypertension & $24(36.9)$ & $153(33.1)$ & 0.54 \\
\hline Diabetes & $7(10.8)$ & $21(4.5)$ & 0.072 \\
\hline Hypercholesterolemia & $23(35.4)$ & $166(35.9)$ & 0.93 \\
\hline Smoking & $41(63.1)$ & $310(67.1)$ & 0.52 \\
\hline Obesity (BMI > 30 kg/m²) & $15(23.1)$ & $60(13)$ & 0.029 \\
\hline Prior Ml & $3(4.6)$ & $30(6.5)$ & 0.76 \\
\hline Prior $\mathrm{PCl}$ & $0(0)$ & $3(0.6)$ & 0.82 \\
\hline Prior CABG & $2(3.1)$ & $20(4.3)$ & 0.89 \\
\hline Atrial fibrillation & $0(0)$ & $6(1.3)$ & 0.76 \\
\hline Heart rate $[\mathrm{bpm}]$ & $81 \pm 17$ & $78 \pm 17$ & 0.18 \\
\hline Cardiac arrest & $4(6.2)$ & $22(4.8)$ & 0.86 \\
\hline \multicolumn{4}{|l|}{ Infarct location } \\
\hline Anterior & $29(44.6)$ & $182(39.4)$ & 0.42 \\
\hline Inferior & $31(47.7)$ & $225(48.7)$ & 0.88 \\
\hline \multicolumn{4}{|l|}{ Onset-to-door time [h] } \\
\hline $0-3$ & $23(37.1)$ & $168(38.9)$ & 0.79 \\
\hline 3-12 & $26(41.9)$ & 168 (38.9) & 0.65 \\
\hline$>12$ & $13(21)$ & $96(22.2)$ & 0.82 \\
\hline \multicolumn{4}{|l|}{ Killip class } \\
\hline 4 & $6(9.2)$ & $17(3.7)$ & 0.084 \\
\hline 3 & $0(0)$ & $1(0.2)$ & 0.25 \\
\hline 1 or 2 & $59(90.8)$ & $444(96.1)$ & 0.11 \\
\hline \multicolumn{4}{|l|}{ LVEF [\%] } \\
\hline$>50$ & $28(75.7)$ & $165(60.2)$ & 0.069 \\
\hline $30-50$ & $7(18.9)$ & $105(38.3)$ & 0.021 \\
\hline$<30$ & $2(5.4)$ & $4(1.5)$ & 0.32 \\
\hline \multicolumn{4}{|l|}{ Number of arteries diseased } \\
\hline 0 & $5(10.4)$ & $50(16.4)$ & 0.29 \\
\hline 1 & $32(66.7)$ & $187(61.3)$ & 0.48 \\
\hline 2 & $7(14.6)$ & $52(17)$ & 0.67 \\
\hline$\geq 3$ & $4(8.3)$ & $16(5.2)$ & 0.60 \\
\hline \multicolumn{4}{|c|}{ Onset-to-reperfusion times [min] } \\
\hline Onset-to-lysis & $225(162-382)$ & $127(60-360)$ & 0.67 \\
\hline Onset-to-balloon & $232(180-358)$ & $235(179-370)$ & 0.78 \\
\hline
\end{tabular}

$B M I$ - body mass index, MI - myocardial infarction, $P C l$ - percutaneous coronary intervention, CABG - coronary artery bypass grafting, LVEF - left ventricular ejection fraction

istration of acetylsalicylic acid, clopidogrel, heparin, $\beta$ - and calcium channel blockers, statins and angiotensin-converting enzyme inhibitors both on admission and after discharge from hospital. The complication rate was very low and not significantly different between men and women (Table IV).

\section{Prognosis}

In-hospital and 30-day mortality were very low and therefore insignificant. However, the tendency of higher mortality in women started very early after discharge from hospital, became significant 
Table II. Therapeutic approach

\begin{tabular}{|lccc|}
\hline Variable & $\begin{array}{c}\text { Females } \\
n=65(\%)\end{array}$ & $\begin{array}{c}\text { Males } \\
n=462(\%)\end{array}$ & Value of $p$ \\
\hline Medical therapy & $15(23.1)$ & $135(29.2)$ & 0.30 \\
\hline $\begin{array}{l}\text { Coronary } \\
\text { angiography }\end{array}$ & $48(73.9)$ & $305(66.0)$ & 0.21 \\
\hline Anti-GP IIb/IIla & $15(23.1)$ & $94(20.3)$ & 0.81 \\
\hline Thrombolysis & $4(6.2)$ & $28(6.1)$ & 0.77 \\
\hline PCI & $40(61.5)$ & $242(52.4)$ & 0.17 \\
\hline
\end{tabular}

$G P$ - glycoprotein, $P C l$ - percutaneous coronary intervention

Table IV. In-hospital complications

\begin{tabular}{|lccc|}
\hline Variable & $\begin{array}{c}\text { Females } \\
n=65(\%)\end{array}$ & $\begin{array}{c}\text { Males } \\
n=462(\%)\end{array}$ & Value of $p$ \\
\hline STEMI & $2(3.1)$ & $15(3.2)$ & 0.76 \\
\hline NSTEMI/UA & $2(3.1)$ & $5(1.1)$ & 0.46 \\
\hline Stroke & $1(1.5)$ & $0(0)$ & 0.25 \\
\hline Bleeding & $0(0)$ & $0(0)$ & - \\
\hline Re-PCI (TLR) & $1(2.5)$ & $0(0)$ & 0.30 \\
\hline
\end{tabular}

STEMI - ST-segment elevation myocardial infarction, NSTEMI - non-STsegment elevation myocardial infarction, UA - unstable angina, $P C I$ - percutaneous coronary intervention, $T L R$-target lesion revascularization

in the sixth month and continued to be significantly higher until the end of the observed period (Table V, Figure 1). In the multivariate analysis three factors were found to influence mortality - Killip class 3 or 4 on admission (RR 75.2, 95\% Cl 19.6-288.4), age per 5-year increase (RR 1.93, 95\% Cl 1.01-3.73), and primary $\mathrm{PCl}$ (RR $0.31,95 \% \mathrm{Cl} 0.1-0.93)$ - whereas all other factors included, as well as female sex, remained insignificant (Figure 2). When analyzing only patients who received reperfusion therapy (either $\mathrm{PCl}$ or fibrinolysis) there was a slight tendency towards higher mortality in women $(7.1 \%$ vs. $1.9 \% ; p=0.046$ ) (Figure 3).

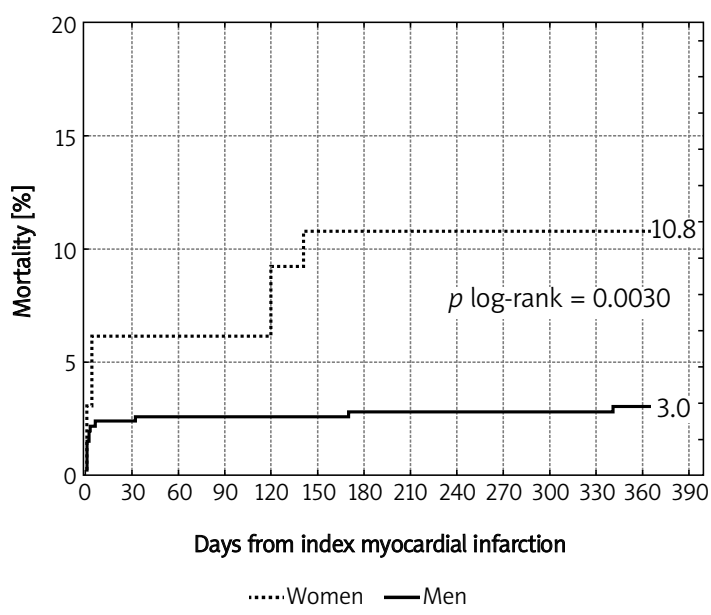

Figure 1. Kaplan-Meier 1-year mortality curves in men and women
Table III. Pre- and postprocedural TIMI flow in patients who underwent PCl

\begin{tabular}{|lccc|}
\hline Variable & $\begin{array}{c}\text { Females } \\
n=40(\%)\end{array}$ & $\begin{array}{c}\text { Males } \\
n=242(\%)\end{array}$ & Value of $p$ \\
\hline \begin{tabular}{l} 
Preprocedural TIMI flow \\
\hline 0
\end{tabular} & & \\
\hline 1 & $7(17.5)$ & $28(11.6)$ & 0.31 \\
\hline 2 & $5(12.5)$ & $27(11.2)$ & 0.96 \\
\hline 3 & $5(12.5)$ & $33(13.6)$ & 0.88 \\
\hline Postprocedural TIMI flow & & \\
\hline 0 & $2(5)$ & $3(1.2)$ & 0.30 \\
\hline 1 & $0(0)$ & $3(1.2)$ & 0.89 \\
\hline 2 & $1(2.5)$ & $14(5.8)$ & 0.65 \\
\hline 3 & $37(92.5)$ & $222(91.7)$ & 0.85 \\
\hline
\end{tabular}

TIMI - Thrombolysis in Myocardial Infarction

Table V. Mortality in men and women

\begin{tabular}{|lccc|}
\hline Variable & $\begin{array}{c}\text { Females } \\
n=65(\%)\end{array}$ & $\begin{array}{c}\text { Males } \\
n=462(\%)\end{array}$ & Value of $p$ \\
\hline In-hospital & $2(3.1)$ & $7(1.5)$ & 0.69 \\
\hline 30-day & $4(6.2)$ & $11(2.4)$ & 0.19 \\
\hline 6-month & $7(10.8)$ & $13(2.8)$ & 0.0052 \\
\hline 12-month & $7(10.8)$ & $14(3.0)$ & 0.003 \\
\hline
\end{tabular}

\section{Discussion}

At the beginning of the $20^{\text {th }}$ century in-hospital mortality in myocardial infarction reached $25-30 \%$. Wide implementation of acute cardiac care units, fibrinolysis and primary $\mathrm{PCl}$ resulted in a 30-day mortality decrease to $8.4 \%$. In large randomized controlled trials on fibrinolysis or primary $\mathrm{PCl}$, 30-day mortality varies from $4 \%$ to $6 \%$ [14]. Despite the progress, STEMI is one of the leading causes of mortality. In Poland, mortality after STEMI has decreased over the last decades, which is strongly associated with the progress in the management of patients with ACS, especially with a markedly developed network of primary $\mathrm{PCl}$ capable catheterization laboratories and modern pharmacotherapy (thienopyridines, glycoprotein IIb/IIla inhibitors, renin-angiotensin-aldosterone system inhibitors, statins and $\beta$-blockers) [15]. Young women are usually believed to have a lower cardiovascular risk as they are under the protective influence of estrogens. Gender-related differences in the management and prognosis have been widely documented in STEMI patients. In general, women with STEMI are older and have more cardiovascular risk factors and are also more prone to complications both of the disease and treatment [9]. There are some conflicting reports of either no association of early mortality with sex and age [16], a worse prognosis only 


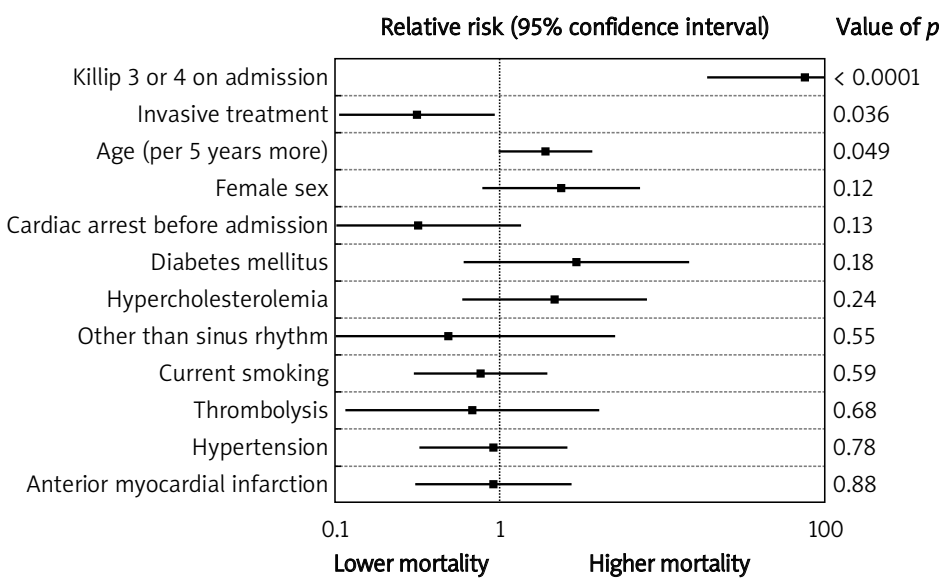

Figure 2. One-year mortality - a multivariate analysis

for older women [11] or only in younger women [17] after myocardial infarction. Studies investigating long-term mortality after STEMI with reference to menopausal status are lacking.

Acute myocardial infarction in young persons represents from $2 \%$ to $10 \%$ of all ACS cases [18] and usually affects males with a high disproportion to females, which is consistent with our findings. A disproportionate number of females may raise concern about data completeness or selection bias and therefore about the strength of the conclusions. In our observation the ratio of females to males is about $1: 7$, which is consistent with findings presented by others or is even more in favor of the female subgroup. Despite the fact that STEMI should be considered as a secondary one to another underlying disease typical for this subset of patients, the cause may be coronary atherosclerosis [2]. The most common risk factor in young patients is smoking, with much higher prevalence than in an older group [19]. Poor basic clinical characteristics of women with STEMI (significantly more frequent hypertension, obesity, diabetes, anterior $\mathrm{MI}$, atrial fibrillation, cardiogenic shock or pulmonary edema and longer onset-to-reperfusion time than in men) are reported in numerous papers as an explanation of the unfavorable prognosis in women. The above statements are true for any population with STEMI but without an age restriction. The question is whether these explanations fully satisfy the reader in the context of the present study results. In our group women had the classical coronary risk factor profile similar to their male counterparts, except for more frequent obesity. In a few reports there were some important findings that younger patients have more frequent history of parental coronary artery disease or a specific lipid phenotype [20, 21]. In the study group the incidence of some manifestations of CAD prior to the index ACS was very low. Unfortunately, data concerning parental history of CAD have not been collected.

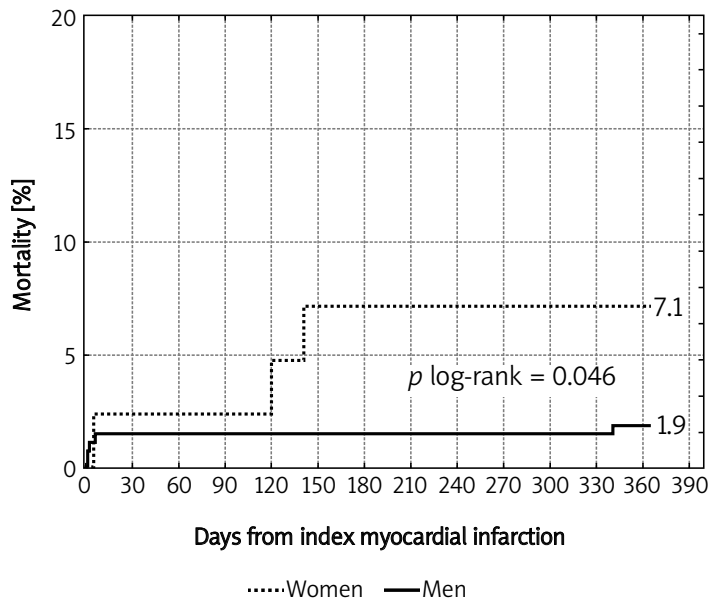

Figure 3. Kaplan-Meier 1-year mortality curves in patients who underwent reperfusion

There was no difference in the prevalence of hypercholesterolemia. Another finding of ours, confirmed by others [19], is that more than $60 \%$ of younger patients were current smokers. When compared to all patients included in the registry [22], it is three times higher in women and almost twice as high in men, but still significant $(p<0.001$; data not presented here). In a multivariate analysis smoking did not influence long-term mortality, but two other factors (cardiogenic shock and age) were much more powerful. Although the beneficial effect of immediate blood flow restoration is certain, in many registries [9] women are less likely to receive reperfusion therapy within a suggested timeframe, which results in a longer total ischemic time, larger extent of necrosis and heart failure, and therefore in a worse long-term prognosis. In our population there were no significant differences between men and women in time delays from symptoms onset to reperfusion. Moreover, the extent of the disease (single vs. multivessel disease) and a high post-procedural success rate (TIMI 3 flow) were similarly distributed within genders. What is most important, 
the majority of women (73.9\%) had angiography and most of them $\mathrm{PCl}$ performed. Even the $\mathrm{PCl}$ to angiography ratio, usually greater in males due to lower PCl eligibility in women (smaller vessel size and greater tortuousness, pre-procedural TIMI 3 flow), in our study was greater in women, which excludes a referral bias. There were no significant differences in adjunctive and post-discharge pharmacotherapy as well as in in-hospital complication rates between genders. Some authors, although not supported by others' findings, ascribe the risk of higher in-hospital mortality in women to a greater incidence of pre-hospital cardiac deaths (therefore not included in registries) in men [9]. Even if this is correct, it only explains early mortality. In our study the incidence of pre- and in-hospital cardiac arrest was insignificant and did not influence mortality in multivariate analysis.

To further explore reasons for higher mortality in women, it would be useful to investigate the correlation between some prognostic parameters (e.g. LVEF) and mortality, as it is commonly known that prognosis after STEMI is poor in individuals with significantly decreased LVEF [14]. Surprisingly, males had a moderately decreased LVEF (30-50\%) more frequently than females $(38.3 \%$ vs. $18.9 \%$; $p=0.021)$, whereas there were no differences in the frequency of normal (>50\%) and significantly decreased $(<30 \%)$ LVEF.

Doubtless a prompt restoration of the blood flow in an infarct-related artery preferably by primary $\mathrm{PCl}$ followed by appropriate pharmacotherapy is beneficial in all eligible patients. Even in the subgroup of patients who underwent acute reperfusion (either fibrinolysis or $\mathrm{PCl}$ ) the mortality rate in women is higher. The most important finding of our analysis is that women under forty affected by STEMI have higher long-term mortality than their male counterparts regardless of the management strictly according to guidelines. In a study by Fournier et al. [18] the overall mortality rate of 104 patients having STEMI under the age of 40 was $25.5 \%$ at 15 years, which confirms findings that individuals younger than forty who are STEMI survivors have a very poor long-term prognosis. In the study by Lawesson et al. [23] in a large cohort of 2132 patients aged below 46 years, women had higher inhospital but not long-term mortality when compared to men even though women had a worse clinical profile and their management was not strictly adherent to guidelines. This is completely opposite to our findings. However, there were some differences in post-discharge pharmacotherapy regarding $\beta$-blockers and statins as well as in the rate of post-discharge NYHA class IV in favor of men in Lawesson's study.

It is emphasized in many papers that crude gender-related differences in mortality depend on clin- ical presentation; however, these differences become less evident or even disappear after adjustment for clinical variables and angiographic findings [24]. What is the most reliable explanation of the present study results that despite no difference in baseline characteristics and management according to guidelines mortality in women, even in those who underwent acute reperfusion, is still significantly higher? Twenty years after Healy's publication [25] Dr C. Merz wrote in her editorial [26] that "the Yentl syndrome is alive and well". So far, we have no proof to question this thesis.

Our registry, as all other registries, has some flaws. One of them is no registration of cause or type of STEMI (e.g. vulnerable plaque disruption vs. cocaine use etc.) and a lipid profile (except presence or absence of hypercholesterolemia), which would be of great importance in this subset of patients. Another problem is a disproportionate number of females as well as a low number of patients, especially the number of deaths during follow-up, which may bias the strength of statistical analyses and, therefore, the conclusions. Unfortunately, all authors reporting gender-related differences within the young with STEMI have the same difficulty. This is a result of the specific characteristics of this particular subset of patients. Therefore, the conclusions of our study should be adjusted to this limitation. To fully clarify the relationship of mortality to an index of myocardial infarction, it would be necessary to collect and analyze all causes of death. However, it was impossible to conduct such analyses. Finally, no data on parental history of premature $\mathrm{MI}$ or the extent of CAD have been collected.

In conclusion, in regard to the limitations considered above, borderline differences in some cardiovascular risk factors that could affect prognosis and low number of patients in the female group, it should be emphasized that any conclusions should be interpreted with caution and only in the context of the present study without extrapolation to the general population. Nonetheless, we conclude that despite almost similar baseline characteristics, no difference in time to reperfusion and even more guideline-compliant management in the population under forty, women with STEMI have higher longterm mortality than their male counterparts. This may question the myth that women are fully protected by estrogens and encourage further investigation of the problem of STEMI in very young people as well as intensification of primary prevention in this population.

\section{References}

1. Stramba-Badiale M, Fox KM, Priori SG, et al. Cardiovascular diseases in women: a statement from the policy conference of the European Society of Cardiology. Eur Heart J 2006; 27: 994-1005. 
2. Cengel A, Tanindi A. Myocardial infarction in the young J Postgrad Med 2009; 55: 305-13.

3. Sakowicz A, Fendler W, Lelonek M, Pietrucha T. Genetic variability and the risk of myocardial infarction in Poles under 45 years of age. Arch Med Sci 2010; 6: 160-7.

4. Hasdai D, Behar S, Wallentin L, et al. A prospective survey of the characteristics, treatments and outcomes of patients with acute coronary syndromes in Europe and the Mediterranean basin; the Euro Heart Survey of Acute Coronary Syndromes (Euro Heart Survey ACS). Eur Heart J 2002; 23: 1190-201.

5. Rosengren A, Wallentin L, K Gitt A, et al. Sex, age, and clinical presentation of acute coronary syndromes. Eur Heart J 2004; 25: 663-70.

6. Vaccarino V, Rathore SS, Wenger NK, et al.; National Registry of Myocardial Infarction Investigators. Sex and racial differences in the management of acute myocardial infarction, 1994 through 2002. N Engl J Med 2005; 353: 671-82.

7. Perers E, Caidahl K, Herlitz J, et al. Spectrum of acute coronary syndromes: history and clinical presentation in relation to sex and age. Cardiology 2004; 102: 67-76.

8. Merz CN, Shaw LJ, Reis SE. Insights from the NHLBISponsored Women's Ischemia Syndrome Evaluation (WISE) Study: Part II: gender differences in presentation, diagnosis, and outcome with regard to gender-based pathophysiology of atherosclerosis and macrovascular and microvascular coronary disease. J Am Coll Cardiol 2006; 47: S21-9.

9. Simon T, Mary-Krause M, Cambou JP, et al.; USIC Investigators. Impact of age and gender on in-hospital and late mortality after acute myocardial infarction: increased early risk in younger women: results from the French nation-wide USIC registries. Eur Heart J 2006; 27: 1282-8.

10. Shavelle DM, Rasouli ML, Frederick P, et al.; National Registry of Myocardial Infarction Investigators. Outcome in patients transferred for percutaneous coronary intervention (a national registry of myocardial infarction 2/3/4 analysis). Am J Cardiol 2005; 96: 1227-32.

11. Marrugat J, Gil M, Masiá R, et al. Role of age and sex in short-term and long term mortality after a first $\mathrm{Q}$ wave myocardial infarction. J Epidemiol Community Health 2001; 55: 487-93.

12. Poloński L, Gąsior M, Gierlotka M, et al. Polish Registry of Acute Coronary Syndromes (PL-ACS). Characteristics, treatments and outcomes of patients with acute coronary syndromes in Poland. Kardiol Pol 2007; 65: 861-72.

13. http://www.rejestrozw.pl. Accessed February 11, 2012.

14. Van de Werf F, Bax J, Betriu A, et al.; ESC Committee for Practice Guidelines (CPG). Management of acute myocardial infarction in patients presenting with persistent ST-segment elevation: the Task Force on the Management of ST-Segment Elevation Acute Myocardial Infarction of the European Society of Cardiology. Eur Heart J 2008; 29: 2909-45.

15. Janion M, Polewczyk A, Gasior M, et al. Progress in the management of ST-segment elevation myocardial infarction during economic transition in Poland between 1992 and 2006. Int J Cardiol 2009; 135: 263-5.

16. Gottlieb S, Harpaz D, Shotan A, et al. Sex differences in management and outcome after acute myocardial infarction in the 1990s: a prospective observational community-based study. Israeli Thrombolytic Survey Group. Circulation 2000; 102: 2484-90.

17. Vaccarino V, Parsons L, Every NR, et al. Sex-based differences in early mortality after myocardial infarction.
National Registry of Myocardial Infarction 2 Participants. N Engl J Med 1999; 22: 217-25.

18. Fournier JA, Cabezón S, Cayuela A, et al. Long-term prognosis of patients having acute myocardial infarction when $</=40$ years of age. Am J Cardiol 2004; 94: 989-92.

19. Teixeira M, Sá I, Mendes JS, et al. Acute coronary syndrome in young adults. Rev Port Cardiol 2010; 29: 947-55.

20. Cuomo S, Guarini P, Gaeta G, et al. Increased carotid intima-media thickness in children-adolescents, and young adults with a parental history of premature myocardial infarction. Eur Heart J 2002; 23: 1345-50.

21. Wiesbauer F, Blessberger H, Azar D, et al. Familial-combined hyperlipidaemia in very young myocardial infarction survivors ( $<$ or $=40$ years of age). Eur Heart J 2009; 30: 1073-9.

22. Sadowski M, Gasior M, Gierlotka M, et al. Clinical characteristics of Polish women with ST-segment elevation myocardial infarction. Kardiol Pol 2010; 68: 627-34.

23. Lawesson SS, Stenestrand U, Lagerqvist B, et al. Gender perspective on risk factors, coronary lesions and longterm outcome in young patients with ST-elevation myocardial infarction. Heart 2010; 96: 453-9.

24. Berger JS, Elliott L, Gallup D, et al. Sex differences in mortality following acute coronary syndromes. JAMA 2009; 302: 874-82.

25. Healy B. The Yentl syndrome. N Engl J Med 1991; 325: 274-6.

26. Merz CN. The Yentl syndrome is alive and well. Eur Heart J 2011; 32: 1313-5. 